\title{
Inflammation-Induced Expression of the Alarmin Interleukin 33 Can Be Suppressed by Galacto-Oligosaccharides
}

\author{
Kim A.T. Verheijden ${ }^{b}$ Peyman Akbari ${ }^{a}{ }^{b}$ Linette E.M. Willemsen ${ }^{b}$ \\ Aletta D. Kraneveld ${ }^{b}$ Gert Folkerts $^{\text {b }}$ Johan Garssen ${ }^{b, c}$ \\ Johanna Fink-Gremmels ${ }^{\text {a }}$ Saskia Braber ${ }^{a}$ \\ ${ }^{a}$ Division of Veterinary Pharmacy, Pharmacology and Toxicology, Utrecht University, ${ }^{b}$ Division of Pharmacology, \\ Utrecht Institute for Pharmaceutical Sciences, Faculty of Science, Utrecht University and 'Nutricia Research, Utrecht, \\ The Netherlands
}

\section{Key Words}

Asthma model · Galacto-oligosaccharides · House-dust mite $\cdot$ Immunomodulation $\cdot$ Interleukin $33 \cdot$ Intestinal permeability

\footnotetext{
Abstract

Background: The alarmin interleukin 33 (IL-33) and its receptor ST2 play an important role in mucosal barrier tissues, and seem to be crucial for Th2-cell mediated host defense. Galacto-oligosaccharides (GOS), used in infant formulas, exhibit gut and immune modulatory effects. To enhance our understanding of the immunomodulatory capacity of GOS, this study investigated the impact of dietary GOS intervention on IL-33 and ST2 expression related to intestinal barrier dysfunction and asthma. Methods: $\mathrm{B} 6 \mathrm{C}_{3} \mathrm{~F}_{1}$ and $\mathrm{BALB} / \mathrm{c}$ mice were fed a control diet with or without $1 \%$ GOS. To simulate intestinal barrier dysfunction, $\mathrm{B}_{6} \mathrm{C}_{3} \mathrm{~F}_{1}$ mice received a gavage with the mycotoxin deoxynivalenol (DON). To mimic asthma-like inflammatory airway responses, BALB/C mice
}

were sensitized on day 0 and challenged on days 7-11 with house-dust mite (HDM) allergen. Samples from the intestines and lungs were collected for IL-33 and ST2 analysis by qRT-PCR, immunoblotting and immunohistochemistry. Results: Dietary GOS counteracted the DON-induced IL-33 mRNA expression and changed the IL-33 distribution pattern in the mouse small intestine. The IL-33 mRNA expression was positively correlated to the intestinal permeability. A strong positive correlation was also observed between IL33 mRNA expression in the lung and the number of bronchoalveolar fluid cells. Reduced levels of IL-33 protein, altered IL-33 distribution and reduced ST2 mRNA expression were observed in the lungs of HDM-allergic mice after GOS intervention. Conclusions: Dietary GOS mitigated IL-33 at the mucosal surfaces in a murine model for intestinal barrier dysfunction and HDM-induced asthma. This promising ef-

Kim A.T. Verheijden and Peyman Akbari contributed equally to this manuscript.

\section{KARGER 125}

(c) 2015 S. Karger AG, Base

$1018-2438 / 15 / 1672-0127 \$ 39.50 / 0$

E-Mail karger@karger.com

www.karger.com/iaa
Correspondence to: Dr. Saskia Braber

Institute for Risk Assessment Sciences, Utrecht University Yalelaan 104

NL-3584 CM Utrecht (The Netherlands)

E-Mail S.Braber@uu.nl 
fect may open up new avenues to use GOS not only as a prebiotic in infant nutrition, but also as a functional ingredient that targets inflammatory processes and allergies associated with IL-33 expression.

(c) 2015 S. Karger AG, Basel

\section{Introduction}

Nondigestible oligosaccharides, such as galacto-oligosaccharides (GOS) and fructo-oligosaccharides (FOS), are currently added to infant milk formulas to achieve an intestinal microbiota composition more similar to that of breastfed infants [1]. Experimental evidence clearly indicates that infants given infant formula enriched with oligosaccharides, particularly GOS, show a significant increase in bifidobacteria and lactobacilli in the microbiota, resembling that of breastfed infants. From other studies, we know that the growth of pathogens is reduced [2]. Besides their effects on the intestinal flora, oligosaccharides can also modulate the activity of the immune system and regulate natural immune mechanisms [3]. Interestingly, a reduction in the incidence of allergic manifestations and infections was observed after nutritional application with prebiotic oligosaccharides (90\% GOS/10\% lcFOS) [4, 5]. Recent work by our group demonstrated that GOS also have microbiota-independent properties observed in intestinal epithelial cells $[6,7]$. The homeostasis of the epithelial inflammatory response within the intestinal epithelium can be regulated by the axis of interleukin 33 (IL33) and its receptor ST2, which belongs to the IL-1/ Toll-like receptor (TLR) superfamily [8]. In recent years, scientific interest in IL-33 has grown, since this cytokine seems to be an indicator of Th2-mediated host defense and plays an important role in mucosal barrier tissues like the intestine and the surface of the airways, where it functions as an endogenous danger signal in response to tissue damage [9]. Moreover, increased expression of IL-33 and its receptor ST2 has been reported in asthma and ulcerative colitis (UC) patients in association with proinflammatory effects $[8,10,11]$.

Since there are indications that GOS can modulate barrier and immune functions and directly interact with epithelial cells, this study aimed to investigate whether the cytokine IL-33 and its receptor ST2 can be affected by the prebiotic GOS. An acute model for intestinal barrier dysfunction and a house-dust mite (HDM)-induced allergic asthma model with an inflammation-induced expression of IL-33 were used to test our hypothesis. Dietary intervention with GOS mitigated the inflammation- induced expression of the alarmin IL-33 in these 2 murine models, while decreased ST2 mRNA expression was observed in the lungs of HDM-allergic mice fed a GOS diet.

\section{Materials and Methods}

\section{Animal Studies}

All in vivo experiments were conducted in compliance with the guidelines of the Ethical Committee on the use of Laboratory Animals of the Utrecht University (DEC 2012.III.02.012 and 2013. II.01.003). Male B6C3F $\mathrm{F}_{1}$ and male BALB/c mice (6-8 weeks old) obtained from Charles River (Maastricht, The Netherlands) were housed under controlled conditions in standard laboratory cages or biocontained sterile conditions using HEPA-filter Isocages ${ }^{\circledR}$ (Tecniplast, Buguggiate, Italy), respectively. These data were obtained from analyses of samples from recently published studies $[6,12]$.

Animals were fed a control diet (AIN-93G) with or without $1 \%$ v/w GOS (Vivinal ${ }^{\circledR}$ GOS syrup with approximately $59 \%$ galacto-oligosaccharides, $21 \%$ lactose, $19 \%$ glucose and $1 \%$ galactose on $75 \%$ dry matter; FrieslandCampina Domo, Borculo, The Netherlands) from day -14 to day 0 [deoxynivalenol (DON) gavage study] and from day -14 to day 14 (asthma study). The carbohydrates in the Vivinal GOS were compensated isocalorically in the control diet by means of cellulose (for GOS), lactose (for lactose) and dextrose (for glucose). Food and water were provided ad libitum.

For the DON gavage study (online suppl. fig. 1; for all online suppl. material, see www.karger.com/doi/10.1159/000437327), DON (D0156; Sigma, St. Louis, Mo., USA) was administered at a dose of $25 \mathrm{mg} / \mathrm{kg}$ of body weight by a single oral gavage to $\mathrm{B}_{6} \mathrm{C} 3 \mathrm{~F}_{1}$ mice at day 0; control mice received sterile PBS. Six hours after the gavage, the mice were sacrificed by cervical dislocation, and the distal small intestine was collected for mRNA isolation and immunohistochemistry. For the asthma study (online suppl. fig. 1), $\mathrm{BALB} / \mathrm{c}$ mice were intranasally sensitized with $1 \mu \mathrm{g}$ HDM allergen/40 $\mu$ l PBS (Greer Laboratories, Lenoir, N.C., USA) under isoflurane anesthesia on day 0 and intranasally challenged daily on days 7-11 with PBS (control) or $10 \mu \mathrm{g}$ HDM/40 $\mu$ PBS. At day 14, the mice were sacrificed by an intraperitoneal overdose of pentobarbital (600 mg/kg, Nembutal ${ }^{\mathrm{TM}}$, Ceva Santé Animale, Naaldwijk, The Netherlands) and the lungs were collected for mRNA isolation, Western blot analysis and immunohistochemistry.

Fluorescein Isothiocyanate-Dextran Permeability Assay

To assess intestinal permeability changes, the intestinal permeability to $4 \mathrm{kDa}$ fluorescein isothiocyanate (FITC)-dextran (Sigma-Aldrich, St. Louis, Mo., USA) was measured as described previously [13]. Briefly, $2 \mathrm{~h}$ after DON administration, all mice received FITC-dextran ( $500 \mathrm{mg} / \mathrm{kg}$ of body weight) by oral gavage. Four hours later, blood was obtained by heart puncture directly after cervical dislocation, and the appearance of FITC-dextran in the blood serum was measured with a spectrofluorometer (FLUOstar Optima; BMG Labtech, Offenburg, Germany).

\section{Bronchoalveolar Lavage}

The trachea of the mice (asthma model) were cannulated and lungs were lavaged 4 times with $1 \mathrm{ml}$ saline solution $(0.9 \%$ $\mathrm{NaCl}$, at $37^{\circ} \mathrm{C}$ ). The bronchoalveolar lavage fluid (BALF) cells 
were centrifuged at $400 \mathrm{~g}$ for $5 \mathrm{~min}$, and then counted using a Bürker-Türk chamber. Differential cell counts were performed on cytospin preparations stained by DiffQuick (Dade, Düdingen, Switzerland).

\section{$q R T-P C R$}

Gene expression was determined by quantitative (q)RTPCR, as described previously [13]. In brief, samples of the distal small intestine and lung tissue were homogenized in RNA lysis buffer with $\beta$-mercaptoethanol. RNA was extracted using spin columns according to the manufacturer's instructions (Promega, Madison, Wis., USA). cDNA was prepared from $1 \mu \mathrm{g}$ of RNA, using the iScript ${ }^{\mathrm{TM}} \mathrm{cDNA}$ synthesis kit (Bio-Rad, Hercules, Calif., USA). qRT-PCR was performed using the MyIQ single-color real-time PCR detection system (Bio-Rad) with iQSYBR green supermix (Bio-Rad). IL-33 and ST2 primers were derived from the NCBI GenBank and manufactured commercially (Eurogentec, Seraing, Belgium); IL-33: forward 5'-GGTGTGGATGGGAAGAAGCTG-3', reverse $5^{\prime}$-GAGGACTTTTTGTGAAGGACG-3' and ST2 forward 5'-CAAGTAGGACCTGTGTGCCC-3' and reverse: 5'CGTGTCCAACAATTGACCTG-3'. The relative amounts of gene expression were standardized and calculated by the expression of house-keeping control gene ( $\beta$-actin) as an internal standard, using the $2^{-\Delta \Delta \mathrm{Ct}}$ method.

\section{Immunoblotting}

Total protein extracts were prepared as described previously [12]. Equal protein amounts were separated by SDS-PAGE, blotted onto PVDF membranes and analyzed with goat anti-mouse IL-33 (R\&D Systems, Minneapolis, Minn., USA; mouse monoclonal antibody, AF3626, 1:500), rabbit anti-ST2 (Abcam, Cambridge, UK; rabbit polyclonal antibody, ab25877, $1: 1,000)$ or rabbit anti- $\beta$ actin (Cell Signaling, Danvers, Mass., USA; rabbit monoclonal antibody, No. 4970, 1:4,000). Appropriate horseradish peroxidaseconjugated secondary antibodies (Dako, Glostrup, Denmark) were used for detection by enhanced chemiluminescence (Amersham Biosciences, Roosendaal, The Netherlands). The band intensity was acquired by a GS710 calibrated imagine densitometer (Bio-Rad).

\section{Immunohistochemistry}

Immunohistochemistry was performed on formalin-fixed, paraffin-embedded distal small intestine (Swiss roll) and lung tissue, using the IL-33 antibody (R\&D Systems; mouse monoclonal antibody, AF3626, 1:500) or ST2 antibody (Abcam; rabbit polyclonal antibody, ab25877, 1:1,200). For antigen retrieval, the slides were boiled in $10 \mathrm{mM}$ citrate buffer ( $\mathrm{pH}$ 6.0) for $10 \mathrm{~min}$ in a microwave [14]. Digital images were acquired using an Olympus BX50 microscope (Olympus Europa GmbH, Hamburg, Germany) equipped with a Leica 320 digital camera (Leica Microsystems, Wetzlar, Germany). No staining was detected in the negative controls, in which the primary antibody had been omitted (online suppl. fig. 2).

\section{ELISA}

IL-33 and ST2 levels in the BALF were measured by ELISA using the Mouse IL-33 ELISA set (R\&D Systems; DY3626) and Mouse ST2 ELISA set (R\&D Systems; DY1004) according to the manufacturer's instructions.

Dietary Galacto-Oligosaccharides

Mitigate IL-33
Statistical Analysis

Statistical analyses were performed with GraphPad Prism 6.0 (Graphpad, LaJolla, Calif., USA). Differences between groups were statistically determined by using one-way ANOVA followed by Bonferroni's multiple-comparison test. Spearman's rank tests were conducted for analyses of correlation. Results were considered to be statistically significant when $\mathrm{p}<0.05$.

\section{Results}

\section{IL-33 mRNA Expression Correlated with the Intestinal}

Permeability Changes and the Number of BALF Cells

In the murine model for intestinal barrier dysfunction, a strong positive correlation was observed between the IL-33 mRNA expression levels in the mouse distal small intestine and the intestinal permeability to $4 \mathrm{kDa}$ FITC-dextran $\left(\mathrm{r}_{\mathrm{s}}=\right.$ 0.6634, $p=0.002$; fig. 1a). In the HDM-induced asthma model, IL-33 mRNA expression levels in the lung positively correlated with the total number of BALF cells $\left(r_{s}=0.5424\right.$, $p=0.0008$; fig. 1b). Differential analysis of the BALF cells showed an increase in eosinophils. The number of lymphocytes and neutrophils was significantly higher in HDM-allergic mice than in control mice (online suppl. table 1).

\section{Dietary Intervention with GOS Counteracts the}

DON-Induced IL-33 mRNA Expression and Distribution

Pattern in the Distal Part of the Mouse Small Intestine

The prominent increase in IL-33 mRNA expression in the distal small intestine observed after DON gavage was prevented by GOS, since the IL-33 mRNA levels in DONtreated animals fed with a GOS diet were significantly lower than in those given a control diet (fig. 2a).

Immunohistochemical staining confirmed that the IL33 production was increased in the distal small intestine after DON gavage (fig. 2c) compared to the control mice that were fed a control or GOS diet (fig. 2b, d). The most pronounced differences were observed in the epithelial layer around the villi. The GOS diet prevented this DONinduced IL-33 production in the distal small intestine, and a lower number of IL-33-expressing epithelial cells were observed (fig. 2e). Related to the IL-33 mRNA expression, the increased ST2 mRNA expression in the distal small intestine of DON-treated animals was reduced in those that were fed a GOS diet; however, this decrease was not statistically significant (fig. 2f). The ST2 immunohistochemical staining depicted in figure $2 \mathrm{~g}-\mathrm{j}$ showed a strong expression pattern in the cytoplasm of the intestinal epithelial cells, and ST2 was also detected in scattered lamina propria mononuclear cells. Similar patterns of ST2 expression for all experimental groups were observed. 


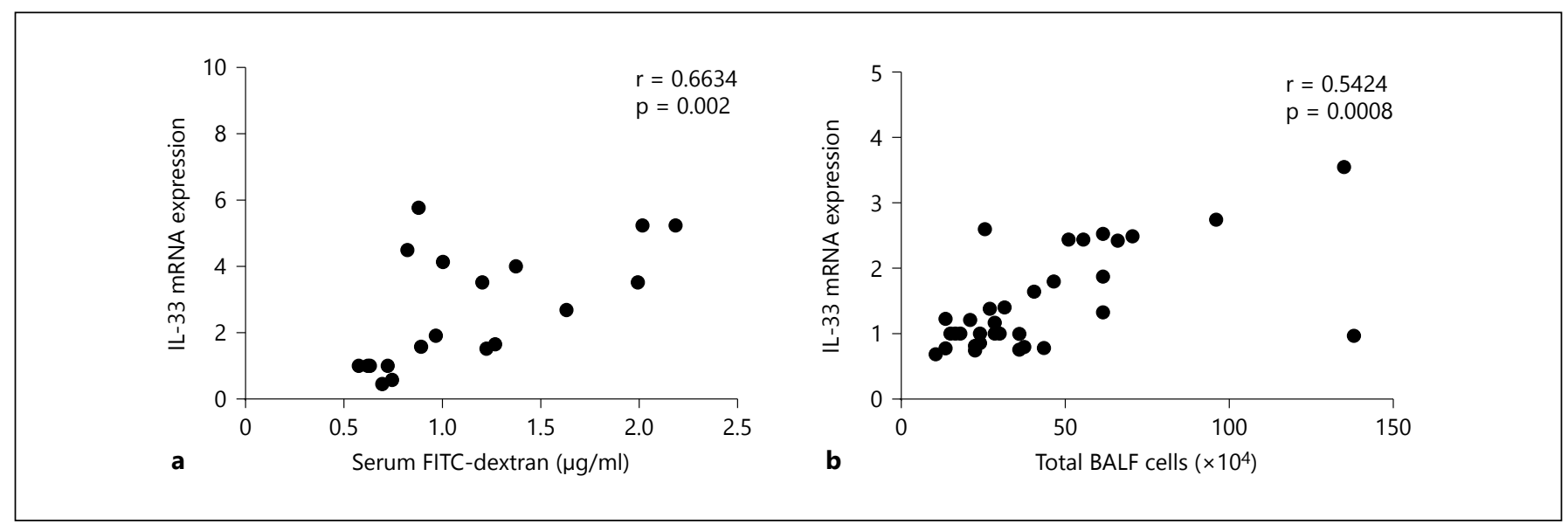

Fig. 1. IL-33 mRNA expression correlated with the intestinal permeability and the number of BALF cells. a Correlation of IL-33 mRNA expression in the distal small intestine and the intestinal permeability to 4 -kDa FITC-dextran in the murine model for
DON-induced intestinal barrier dysfunction. b Correlation of IL33 mRNA expression in the lungs and the total number of BALF cells in the HDM-induced asthma model. Correlation was analyzed using Spearman's correlation test.

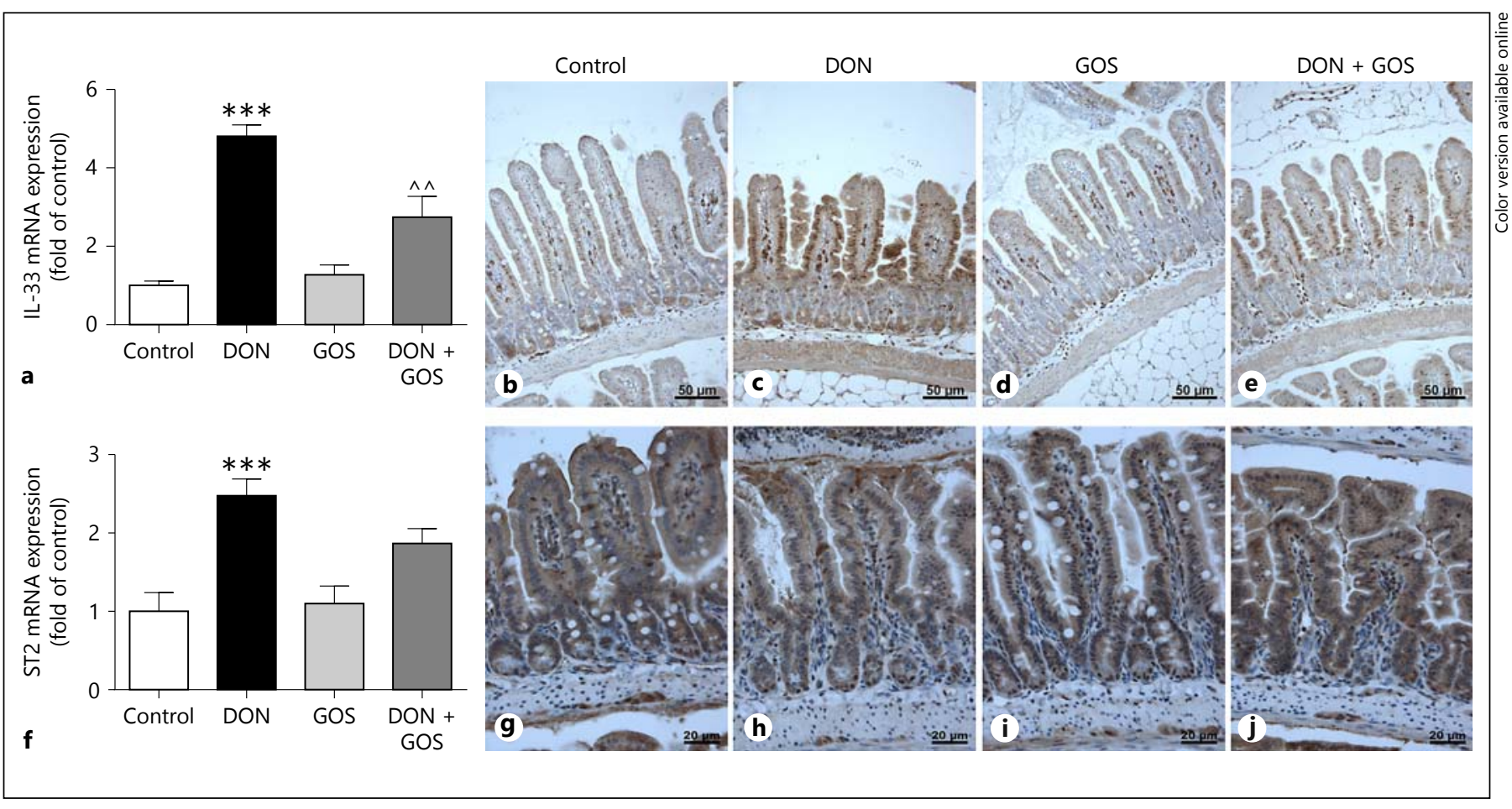

Fig. 2. Dietary intervention with GOS counteracts the DON-induced IL-33 mRNA expression and distribution pattern in the distal part of the mouse small intestine. Mice were fed a control diet or a diet supplemented with GOS for 2 weeks, followed by an oral gavage with DON ( $25 \mathrm{mg} / \mathrm{kg}$ of body weight). a, f Six hours after the DON challenge, the mRNA levels of IL-33 and ST2 were measured by qRT-PCR. Results are expressed as IL-33 mRNA expres- sion (qRT-PCR, normalized to $\beta$-actin) as mean \pm SEM. There were 5-6 animals/experimental group. For immunohistochemistry, Swiss-rolled paraffin sections obtained from distal small intestine were stained with anti-IL-33 (b-e) and anti-ST2 ( $\mathbf{g}-\mathbf{j})$ antibodies (Materials and Methods). ${ }^{* * *} \mathrm{p}<0.001$ significantly different from the control group, $\wedge \wedge \mathrm{p}<0.01$ significantly different from the DON-treated animals. $\times 200$ (IL-33 staining). $\times 400$ (ST2 staining). 


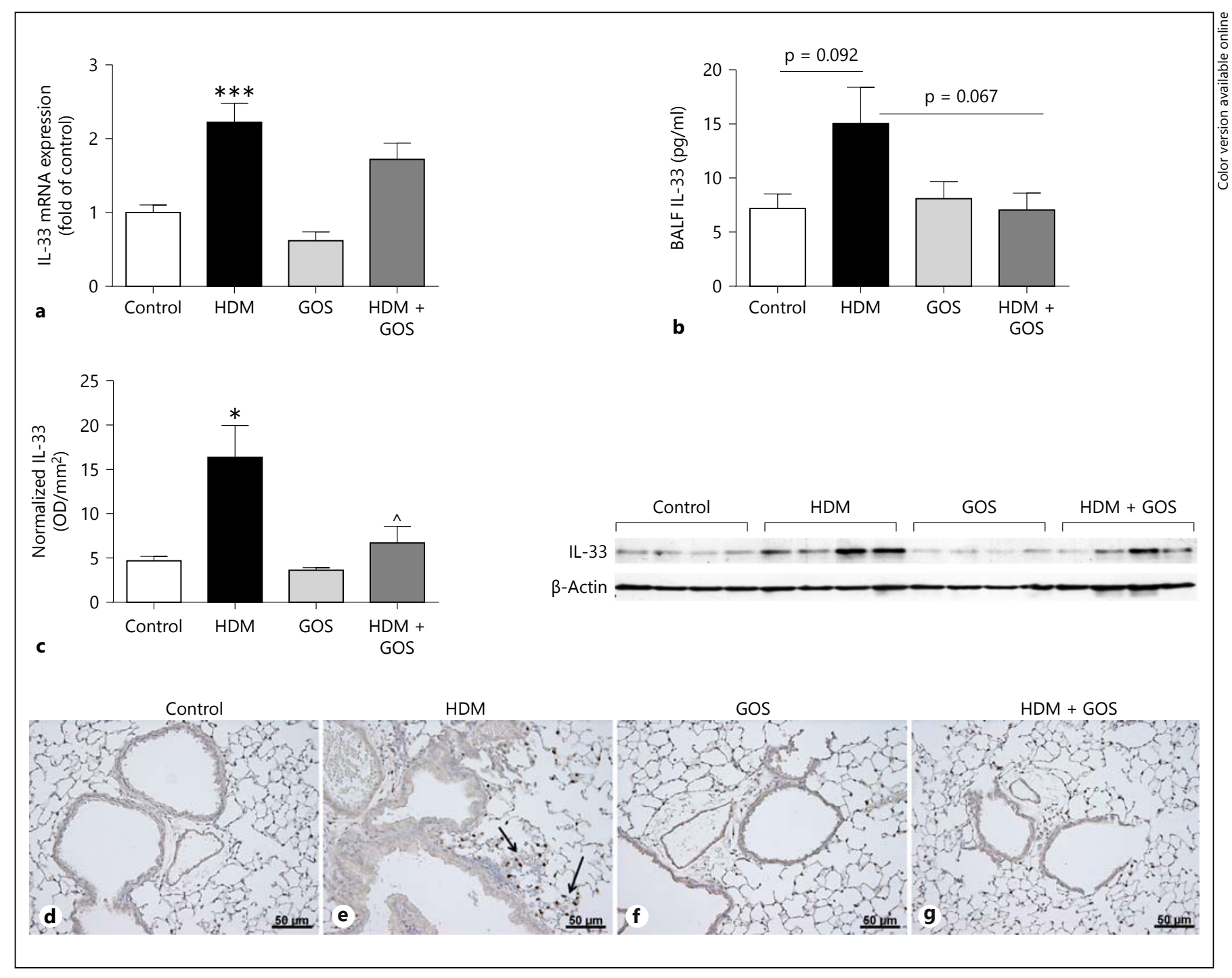

Fig. 3. Dietary intervention with GOS reduces IL-33 protein levels and expression in the lungs of HDM-allergic mice. Mice fed a control diet or a diet supplement with GOS from day -14 to day 14 were sensitized with HDM on day 0 and were challenged on days 7-11 with HDM or PBS (control). IL-33 mRNA expression in the lungs (a), IL-33 concentration in the BALF (b) and IL-33 protein levels in the lungs (c) were measured. Results are expressed as IL-33 mRNA

\section{Dietary Intervention with GOS Reduces IL-33 and}

ST2 mRNA Expression and IL-33 Protein Levels in the Lungs of HDM-Allergic Mice

The increase in IL-33 mRNA expression in the lungs of HDM-allergic mice was partly reduced by dietary intervention with GOS (fig. 3a); however, this decrease was not statically significant. The same trend was observed for the IL-33 concentration in the BALF (fig. 3b). Moreover, the IL-33 protein levels in the lung tissue homogenates of

Dietary Galacto-Oligosaccharides Mitigate IL-33 expression (qRT-PCR, normalized to $\beta$-actin), pg/ml BALF (ELISA) or $\mathrm{OD} / \mathrm{mm}^{2}$ (Western blot, normalized to $\beta$-actin) as mean \pm SEM. There were 7-9 animals/experimental group. For immunohistochemistry, lung sections were stained with anti-IL-33 antibody (dg) as described in Materials and Methods. ${ }^{*} \mathrm{p}<0.05$ and ${ }^{* * *} \mathrm{p}<$ 0.001 significantly different from the control group, $\wedge p<0.05$ significantly different from the HDM-allergic animals. $\times 200$.

GOS-treated HDM-allergic mice were significantly decreased compared to the nontreated HDM-allergic mice (fig. 3c). Comparable with the Western blot data, immunohistochemical staining indicated that more IL-33 expressing cells were present in the lungs of the HDM-allergic mice (fig. 3e) than in the control mice (fed a control or GOS diet; fig. 3d, f). Dietary intervention with GOS caused a decrease in IL-33 expression in the lungs of HDM-allergic mice compared to the nontreated HDM- 


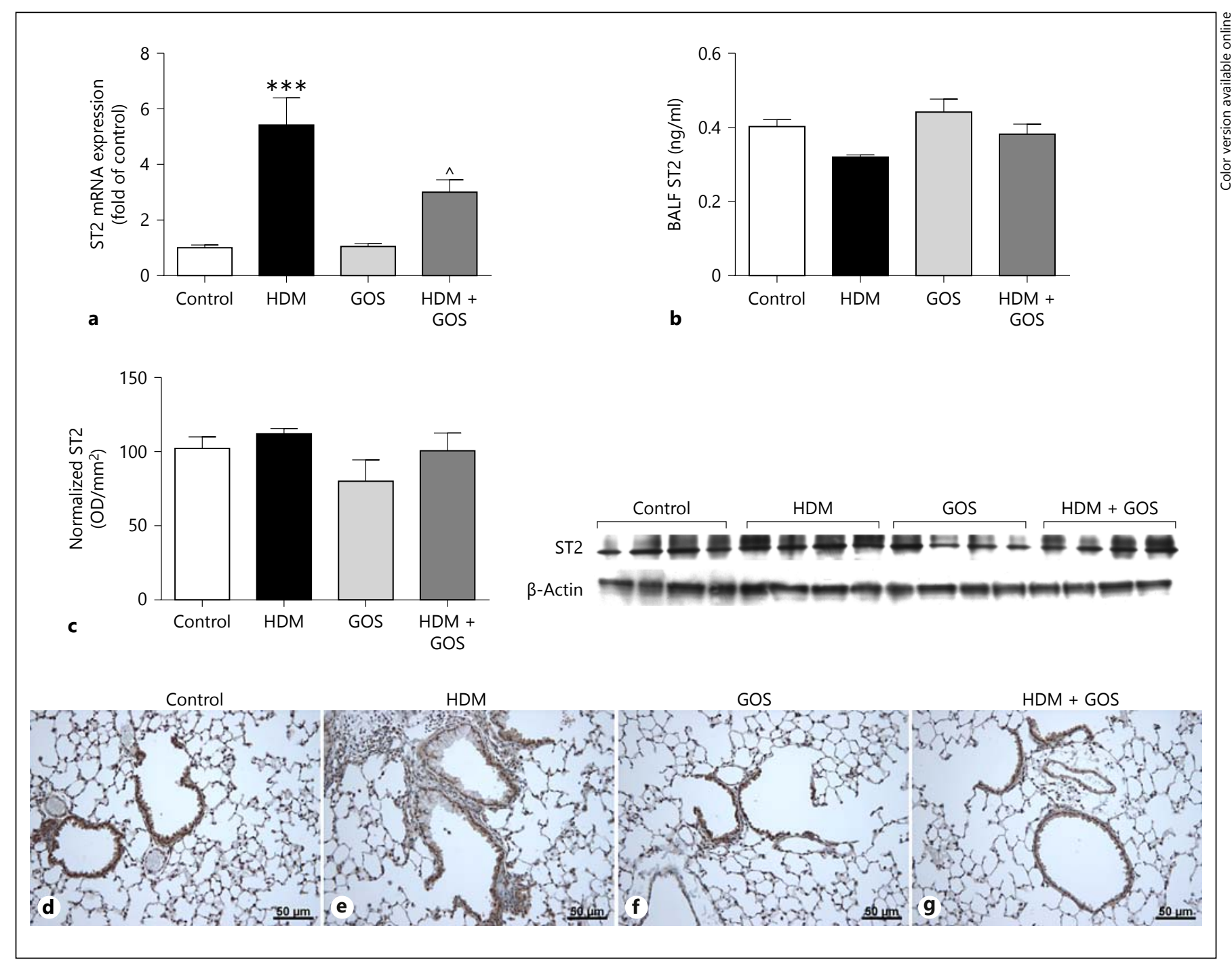

Fig. 4. Dietary intervention with GOS reduces ST2 mRNA expression in the lungs of HDM-allergic mice. Mice fed a control diet or a diet supplement with GOS from day -14 to day 14 were sensitized with HDM on day 0 and were challenged on days 7-11 with HDM or PBS (control). ST2 mRNA expression in the lungs (a), ST2 concentration in the BALF (b) and ST2 protein levels in the lungs (c) were measured and results are expressed as ST2 mRNA expression

allergic mice (fig. 3g). Furthermore, a clear increase in ST2 mRNA expression was observed in the lungs of HDM-allergic mice, which was significantly reduced in the HDM-allergic mice that were fed a GOS diet (fig. 4a). The ST2 levels in the BALF (fig. 4b) and the ST2 protein levels in the lung (fig. 4c) did not significantly differ between the experimental groups; this was confirmed by immunohistochemical staining showing the airway epithelium as a primary source for ST2 (fig. $4 \mathrm{~d}-\mathrm{g}$ ).
(qRT-PCR, normalized to $\beta$-actin), pg/ml BALF (ELISA) or OD/ $\mathrm{mm}^{2}$ (Western blot, normalized to $\beta$-actin) as mean \pm SEM. There were 7-9 animals/experimental group (qRT-PCR and ELISA). For immunohistochemistry, lung sections were stained with anti-ST2 antibody $(\mathbf{d}-\mathbf{g})$ as described in Materials and Methods. ${ }^{* * *} \mathrm{p}<$ 0.001 significantly different from the control group, $\wedge \mathrm{p}<0.05$ significantly different from the HDM-allergic animals. $\times 200$.

\section{Discussion}

IL-33 is a member of the IL-1 cytokine family. It has a dual function: it activates various immune cells through the IL-33 receptor ST2 and acts as an intracellular factor with transcriptional properties [15]. It also delivers an important danger signal in the cellular response to tissue damage, and epithelial cells at mucosal barrier sites constitutively express IL-33 [16]. Specific ef-
Verheijden/Akbari/Willemsen/Kraneveld/ Folkerts/Garssen/Fink-Gremmels/Braber 
fects in the gut can be exerted by IL-33, since mice injected intraperitoneally with recombinant IL-33 have been found to demonstrate an increase in the permeability of the mucosal barrier and intestinal inflammation as well as hypertrophy and hyperplasia of goblet cells $[17,18]$.

In our study, the mycotoxin DON serves as a reliable and reproducible model of intestinal barrier dysfunction $[13,19]$ and a strong positive correlation was observed between IL-33 mRNA expression in the mouse distal small intestine and the intestinal permeability induced by DON. Besides IL-33, the tight and adherens junctions are also critical for the maintenance of intestinal barrier integrity [20-22]. IL-33 impairs the epithelial barrier function; this was observed in a human colonic epithelial Caco-2 monolayer and in mice treated with exogenous IL-33. IL-33 injected intraperitoneally exacerbated sodium (DSS)-induced colitis in mice [23, 24]. Observations in IL-33 ${ }^{-/}$mice suggested that IL-33 deficiency leads to delayed local inflammation and tissue damage during experimental colitis [25]. On the other hand, it was recently published that IL-33 promotes regulatory T-cell function in the intestine. IL-23, an important proinflammatory cytokine in the pathogenesis of irritable bowel disease inhibits this regulatory T-cell responsiveness to IL-33 [26, 27]. Furthermore, IL-33 is found to be prominently present in inflamed colon tissue, mainly localized to the surface epithelium and in the crypt cells, in patients with Crohn's disease and UC $[8,28,29]$. In this study, a similar pattern of distribution of IL-33 was observed in the mouse small intestine after disrupting the intestinal barrier with DON. Besides the increase in IL-33 expression observed by means of immunohistochemical staining, a 5 -fold increase was observed in IL-33 mRNA levels in the mouse distal small intestine after DON gavage, but the levels of other cytokines and chemokines, such as IFN- $\gamma$, IL- $1 \alpha$, IL- $1 \beta$, IL-4, IL- 6 and TNF- $\alpha$ remained unchanged (described by Akbari et al. [6]).

The IL-33/ST2 system plays an important role in irritable bowel disease [29-31]. Pastorelli et al. [28] described that a specific imbalance between IL-33 and ST2 may play a pathogenic role in UC, since ST2 is decreased in UC colonic epithelium and IL-33 is markedly increased in active UC. In this study, no effect of the DON gavage on the ST2 distribution in the distal small intestine was observed; however, the ST2 mRNA expression was significantly increased in the distal small intestine of the DON-treated animals.

The DON-induced increase in IL-33 was mitigated by dietary intervention with GOS, observed in the IL-33
mRNA levels and the distribution of IL-33 in the intestine, but GOS did not affect the DON-induced ST2 expression. Recently, we observed that GOS directly protects the intestinal barrier function by maintaining tight-junction proteins and modulating CXCL8 responses in a human Caco-2 cell monolayer and in a mouse model for intestinal barrier dysfunction. However, the DON-induced hyperpermeability of the intestines for FITC-dextran $(4 \mathrm{kDa})$ was not altered by GOS [6].

As IL-33 is implicated in the Th2-type responses required for the development of allergic inflammation, the effect of dietary GOS on IL-33 expression was also investigated in a murine HDM-induced asthma model. In this model, the IL-33 mRNA expression levels in the lung positively correlated with the number of total BALF cells. Previous studies suggested that IL-33 and ST2 are both associated with the development and maintenance of allergic asthma and are correlated with disease severity [32, 33]. It has been suggested that the IL-33-induced production of proinflammatory cytokines is a critical event that aggravates asthma [34]. Treatments with anti-IL-33 monoclonal antibody have been reported to inhibit allergen-induced airway inflammation, Th2 cytokine production and mucus hypersecretion in mice [35]. Intranasally challenged IL-33-deficient mice showed impaired IL-5 and IL-13 production from group 2 innate lymphoid cells as well as lung inflammation and Th2 cell differentiation $[36,37]$. Administration of blocking anti-ST2 antibodies or ST2-immunoglobulin fusion protein to allergic mice abrogated the Th2-mediated inflammatory response [38]. Contradictory results are described for T1/ST2-deficient mice; Hoshino et al. [39] observed normal Th2 responses in these animals, while Townsend et al. [40] showed reduced levels of IL-4 and IL-5.

In line with the data of the intestinal barrier dysfunction model, dietary intervention with GOS resulted in lower IL-33 and ST2 levels and an altered IL-33 distribution in the lungs of HDM-allergic mice. In addition, the increased concentration of the Th2 cytokine IL-13 in the lung of HDM-allergic mice was significantly decreased by dietary intervention with GOS and the same trend was observed for the IL-5 concentration in the BALF, although this was not significantly different (online suppl. table 2) [12].

The mode of action of GOS is complex and still not entirely understood. The reduced IL-33 expression exerted by GOS might result from alterations in the composition of the microbiota because, initially, GOS was considered as a typical prebiotic, supporting the growth 
of Lactobacillus and Bifidobacterium spp. in the large intestine $[1,41]$. GOS not only stimulates these bacteria, but affects the whole intestinal flora by production of short-chain fatty acids like butyrate and decreasing the $\mathrm{pH}[1,42]$. It is known that butyrate exerts anti-inflammatory properties, which may explain the desirable effects of various oligosaccharides [43]. In turn, different immune-related, anti-allergic and anti-inflammatory effects have been observed in vivo after GOS/lcFOS supplementation, suggesting a positive effect on mucosal immunity via suppression of Th2-type responses, a downregulation of total immunoglobulin levels and an induction of Th1 cell and regulatory $\mathrm{T}$-cell polarization $[4,5,44,45]$.

Moreover, GOS seem to exert direct, microbiota-independent effects on the immune system by interacting with epithelial and immune cells, as indicated by in vitro experiments $[3,6,7,46]$. Although direct interaction with TLR4 has been hypothesized [46], the direct effect of GOS on tight junction assembly in Caco-2 cells [6] indicates the involvement of other mechanisms as well, since Caco2 cells do not express TLR4. Furthermore, different galectins have distinct binding specificities for binding oligosaccharides [47]. Indeed, previous investigations show that dietary GOS enhanced the serum galectin-9 levels, which are involved in the regulation of immune responses and tolerance induction, which leads to a suppression of allergic symptoms in mice and humans [48].

These findings can be considered as a first indication of the systemic modulatory effect of GOS, and they are now supported by our findings that GOS suppresses IL33 , an alarmin that is produced at different mucosal surfaces. The parallel response of intestinal repair mechanisms and the anti-allergic properties of GOS may therefore be attributable to the systemic effects of signaling molecules like galectins and specific cytokines like IL-33. Further research is needed to investigate whether GOS directly interacts with the IL-33/ST2 system or whether it prevents intestinal barrier disruption and allergic asthma by altering the microbiota composition which, indirectly, leads to a decreased IL-33 production.

In conclusion, dietary intervention with GOS mitigated the important immunomodulator IL-33 in mouse intestines, observed in a model for intestinal barrier dysfunction, and also in murine lungs, observed in a HDMinduced asthma model, which is not necessarily associated with ST2 expression. These preclinical experiments warrant studies on clinical relevance and in order to unravel the mechanism behind this effect.

\section{Acknowledgements}

The project was jointly funded by the European Union, European Regional Development Fund and The Ministry of Economic Affairs, Agriculture and Innovation, Peaks in the Delta, the Municipality of Groningen, the Provinces of Groningen, Fryslân and Drenthe, the Dutch Carbohydrate Competence Center (CCC WP25; www.cccresearch.nl), Nutricia Research and FrieslandCampina.

\section{Disclosure Statement}

PA, ADK, GF, LEMW and JFG have no interests to declare. SB and KATV were granted by the Carbohydrate Competence Center (CCC) programme as indicated in acknowledgements; JG is associated with Nutricia Research, which is an industrial partner in the Dutch Carbohydrate Competence Center.

\section{References}

1 Fanaro S, Boehm G, Garssen J, Knol J, Mosca F, Stahl B, Vigi V: Galacto-oligosaccharides and long-chain fructo-oligosaccharides as prebiotics in infant formulas: a review. Acta Paediatr Suppl 2005;94:22-26.

-2 Ben XM, Zhou XY, Zhao WH, Yu WL, Pan W, Zhang WL, Wu SM, Van Beusekom CM, Schaafsma A: Supplementation of milk formula with galacto-oligosaccharides improves intestinal micro-flora and fermentation in term infants. Chin Med J (Engl) 2004;117:927-931.

-3 Jeurink PV, van Esch BC, Rijnierse A, Garssen J, Knippels LM: Mechanisms underlying immune effects of dietary oligosaccharides. Am J Clin Nutr 2013;98:572S-577S.
4 Arslanoglu S, Moro GE, Boehm G: Early supplementation of prebiotic oligosaccharides protects formula-fed infants against infections during the first 6 months of life. J Nutr 2007; 137:2420-2424.

5 van Hoffen E, Ruiter B, Faber J, M'Rabet L, Knol EF, Stahl B, Arslanoglu S, Moro G, Boehm G, Garssen J: A specific mixture of short-chain galacto-oligosaccharides and long-chain fructo-oligosaccharides induces a beneficial immunoglobulin profile in infants at high risk for allergy. Allergy 2009;64:484487.

6 Akbari P, Braber S, Alizadeh A, Verheijden KA, Schoterman MH, Kraneveld AD, Gars- sen J, Fink-Gremmels J: Galacto-oligosaccharides protect the intestinal barrier by maintaining the tight junction network and modulating the inflammatory responses after a challenge with the mycotoxin deoxynivalenol in human Caco-2 cell monolayers and B6C3F1 mice. J Nutr 2015;145:1604-1613.

7 Varasteh S, Braber S, Garssen J, Fink-Gremmels J: Galacto-oligosaccharides exert a protective effect against heat stress in a Caco-2 cell model. J Funct Foods 2015;16:265-277.

8 Garcia-Miguel M, Gonzalez MJ, Quera R, Hermoso MA: Innate immunity modulation by the IL-33/ST2 system in intestinal mucosa. Biomed Res Int 2013;2013:142492. 
9 Cayrol C, Girard JP: IL-33: an alarmin cytokine with crucial roles in innate immunity, inflammation and allergy. Curr Opin Immunol 2014;31C:31-37.

10 Miller AM: Role of IL-33 in inflammation and disease. J Inflamm (Lond) 2011;8:22.

-11 Hamzaoui A, Berraies A, Kaabachi W, Haifa M, Ammar J, Kamel H: Induced sputum levels of IL-33 and soluble ST2 in young asthmatic children. J Asthma 2013;50:803-809.

12 Verheijden KA, Willemsen LE, Braber S, Leusink-Muis T, Delsing DJ, Garssen J, Kraneveld AD, Folkerts G: Dietary galactooligosaccharides prevent airway eosinophilia and hyperresponsiveness in a murine house dust mite-induced asthma model. Respir Res 2015;16:17.

13 Akbari P, Braber S, Gremmels H, Koelink PJ, Verheijden KA, Garssen J, Fink-Gremmels J: Deoxynivalenol: a trigger for intestinal integrity breakdown. FASEB J 2014;28:24142429.

-14 Paulissen G, El Hour M, Rocks N, Gueders MM, Bureau F, Foidart JM, Lopez-Otin C, Noel A, Cataldo DD: Control of allergen-induced inflammation and hyperresponsiveness by the metalloproteinase ADAMTS-12. J Immunol 2012;189:4135-4143.

15 Ali S, Mohs A, Thomas M, Klare J, Ross R, Schmitz ML, Martin MU: The dual function cytokine IL-33 interacts with the transcription factor NF-kappaB to dampen NF-kappaB-stimulated gene transcription. J Immunol 2011;187:1609-1616.

16 Pichery M, Mirey E, Mercier P, Lefrancais E, Dujardin A, Ortega N, Girard JP: Endogenous IL-33 is highly expressed in mouse epithelial barrier tissues, lymphoid organs, brain, embryos, and inflamed tissues: in situ analysis using a novel Il-33-LacZ gene trap reporter strain. J Immunol 2012;188:34883495.

$>17$ Schmitz J, Owyang A, Oldham E, Song Y, Murphy E, McClanahan TK, Zurawski G, Moshrefi M, Qin J, Li X, Gorman DM, Bazan JF, Kastelein RA: IL-33, an interleukin-1-like cytokine that signals via the IL-1 receptor-related protein ST2 and induces T helper type 2 -associated cytokines. Immunity 2005;23: 479-490.

18 Yang Z, Grinchuk V, Urban JF Jr., Bohl J, Sun R, Notari L, Yan S, Ramalingam T, Keegan AD, Wynn TA, Shea-Donohue T, Zhao A: Macrophages as IL-25/IL-33-responsive cells play an important role in the induction of type 2 immunity. PLoS One 2013;8:e59441.

19 Pinton P, Nougayrede JP, Del Rio JC, Moreno C, Marin DE, Ferrier L, Bracarense AP, Kolf-Clauw M, Oswald IP: The food contaminant deoxynivalenol decreases intestinal barrier permeability and reduces claudin expression. Toxicol Appl Pharmacol 2009;237: 41-48.

-20 Groschwitz KR, Hogan SP: Intestinal barrier function: molecular regulation and disease pathogenesis. J Allergy Clin Immunol 2009; 124:3-20; quiz 21-22.
21 Van Itallie CM, Anderson JM: Architecture of tight junctions and principles of molecular composition. Semin Cell Dev Biol 2014;36: 157-165.

22 Niessen CM: Tight junctions/adherens junctions: basic structure and function. J Invest Dermatol 2007; 127:2525-2532.

23 Sedhom MA, Pichery M, Murdoch JR, Foligne B, Ortega N, Normand S, Mertz K, Sanmugalingam D, Brault L, Grandjean T, Lefrancais E, Fallon PG, Quesniaux V, PeyrinBiroulet L, Cathomas G, Junt T, Chamaillard M, Girard JP, Ryffel B: Neutralisation of the interleukin-33/ST2 pathway ameliorates experimental colitis through enhancement of mucosal healing in mice. Gut 2013;62:17141723.

4 Imaeda $\mathrm{H}$, Andoh A, Aomatsu T, Uchiyama K, Bamba S, Tsujikawa T, Naito Y, Fujiyama Y: Interleukin-33 suppresses Notch ligand expression and prevents goblet cell depletion in dextran sulfate sodium-induced colitis. Int J Mol Med 2011;28:573-578.

25 Oboki K, Ohno T, Kajiwara N, Arae K, Morita H, Ishii A, Nambu A, Abe T, Kiyonari $\mathrm{H}$, Matsumoto K, Sudo K, Okumura K, Saito H, Nakae S: IL-33 is a crucial amplifier of innate rather than acquired immunity. Proc Natl Acad Sci U S A 2010;107: 18581-18586.

26 Schiering C, Krausgruber T, Chomka A, Frohlich A, Adelmann K, Wohlfert EA, Pott J, Griseri T, Bollrath J, Hegazy AN, Harrison OJ, Owens BM, Lohning M, Belkaid Y, Fallon PG, Powrie F: The alarmin IL-33 promotes regulatory $\mathrm{T}$-cell function in the intestine. Nature 2014;513:564-568.

27 Matta BM, Lott JM, Mathews LR, Liu Q, Rosborough BR, Blazar BR, Turnquist HR: IL-33 is an unconventional alarmin that stimulates IL-2 secretion by dendritic cells to selectively expand IL-33R/ST2+ regulatory T cells. J Immunol 2014;193:4010-4020.

28 Pastorelli L, Garg RR, Hoang SB, Spina L, Mattioli B, Scarpa M, Fiocchi C, Vecchi M, Pizarro TT: Epithelial-derived IL-33 and its receptor ST2 are dysregulated in ulcerative colitis and in experimental Th1/Th2 driven enteritis. Proc Natl Acad Sci U S A 2010;107: 8017-8022.

29 Seidelin JB, Bjerrum JT, Coskun M, Widjaya B, Vainer B, Nielsen OH: IL-33 is upregulated in colonocytes of ulcerative colitis. Immunol Lett 2010;128:80-85.

30 Beltran CJ, Nunez LE, Diaz-Jimenez D, Farfan N, Candia E, Heine C, Lopez F, Gonzalez MJ, Quera R, Hermoso MA: Characterization of the novel ST2/IL-33 system in patients with inflammatory bowel disease. Inflamm Bowel Dis 2010;16:1097-1107.

31 Kobori A, Yagi Y, Imaeda H, Ban H, Bamba S, Tsujikawa T, Saito Y, Fujiyama Y, Andoh A: Interleukin-33 expression is specifically enhanced in inflamed mucosa of ulcerative colitis. J Gastroenterol 2010;45:999-1007.

32 Lloyd CM: IL-33 family members and asthma - bridging innate and adaptive immune responses. Curr Opin Immunol 2010;22:800806.

33 Oshikawa K, Kuroiwa K, Tago K, Iwahana H, Yanagisawa K, Ohno S, Tominaga SI, Sugiyama Y: Elevated soluble ST2 protein levels in sera of patients with asthma with an acute exacerbation. Am J Respir Crit Care Med 2001; 164:277-281.

34 Nabe T: Interleukin (IL)-33: new therapeutic target for atopic diseases. J Pharmacol Sci 2014;126:85-91.

35 Mizutani N, Nabe T, Yoshino S: Interleukin-33 and alveolar macrophages contribute to the mechanisms underlying the exacerbation of IgE-mediated airway inflammation and remodelling in mice. Immunology 2013; 139:205-218.

36 Kamijo S, Takeda H, Tokura T, Suzuki M Inui K, Hara M, Matsuda $\mathrm{H}$, Matsuda A, Oboki K, Ohno T, Saito H, Nakae S, Sudo K, Suto $\mathrm{H}$, Ichikawa S, Ogawa H, Okumura K, Takai T: IL-33-mediated innate response and adaptive immune cells contribute to maximum responses of protease allergen-induced allergic airway inflammation. J Immunol 2013;190: 4489-4499.

37 Halim TY, Steer CA, Matha L, Gold MJ, Martinez-Gonzalez I, McNagny KM, McKenzie AN, Takei F: Group 2 innate lymphoid cells are critical for the initiation of adaptive T helper 2 cell-mediated allergic lung inflammation. Immunity 2014; 40: 425-435.

38 Coyle AJ, Lloyd C, Tian J, Nguyen T, Erikkson C, Wang L, Ottoson P, Persson P, Delaney T, Lehar S, Lin S, Poisson L, Meisel C, Kamradt T, Bjerke T, Levinson D, Gutierrez-Ramos JC: Crucial role of the interleukin 1 receptor family member T1/ST2 in Thelper cell type 2-mediated lung mucosal immune responses. J Exp Med 1999;190:895-902.

39 Hoshino K, Kashiwamura S, Kuribayashi K, Kodama T, Tsujimura T, Nakanishi K, Matsuyama T, Takeda K, Akira S: The absence of interleukin 1 receptor-related T1/ST2 does not affect $\mathrm{T}$ helper cell type 2 development and its effector function. J Exp Med 1999;190: 1541-1548.

40 Townsend MJ, Fallon PG, Matthews DJ, Jolin HE, McKenzie AN: T1/ST2-deficient mice demonstrate the importance of $\mathrm{T} 1 /$ ST2 in developing primary $\mathrm{T}$ helper cell type 2 responses. J Exp Med 2000;191:10691076.

41 Giovannini M, Verduci E, Gregori D, Ballali S, Soldi S, Ghisleni D, Riva E, for the PTSG: Prebiotic effect of an infant formula supplemented with galacto-oligosaccharides: randomized multicenter trial. J Am Coll Nutr 2014;33:385-393.

42 Holscher HD, Faust KL, Czerkies LA, Litov R, Ziegler EE, Lessin H, Hatch T, Sun S, Tappenden KA: Effects of prebiotic-containing infant formula on gastrointestinal tolerance and fecal microbiota in a randomized controlled trial. JPEN J Parenter Enteral Nutr 2012;36: 95S-105S.
Dietary Galacto-Oligosaccharides

Mitigate IL-33
Int Arch Allergy Immunol 2015;167:127-136 DOI: $10.1159 / 000437327$ 
43 Oozeer R, van Limpt K, Ludwig T, Ben Amor K, Martin R, Wind RD, Boehm G, Knol J: Intestinal microbiology in early life: specific prebiotics can have similar functionalities as human-milk oligosaccharides. Am J Clin Nutr 2013;98:561S-571S.

-44 Vos AP, M'Rabet L, Stahl B, Boehm G, Garssen J: Immune-modulatory effects and potential working mechanisms of orally applied nondigestible carbohydrates. Crit Rev Immunol 2007;27:97-140.
45 Schijf MA, Kruijsen D, Bastiaans J, Coenjaerts FE, Garssen J, van Bleek GM, van't Land B: Specific dietary oligosaccharides increase Th1 responses in a mouse respiratory syncytial virus infection model. J Virol 2012;86:11472-11482.

46 Ortega-Gonzalez M, Ocon B, Romero-Calvo I, Anzola A, Guadix E, Zarzuelo A, Suarez MD, Sanchez de Medina F, Martinez-Augustin O: Nondigestible oligosaccharides exert nonprebiotic effects on intestinal epithelial cells enhancing the immune response via activation of TLR4-NFkappaB. Mol Nutr Food Res 2014;58:384-393.
7 de Kivit S, Kraneveld AD, Garssen J, Willemsen LE: Glycan recognition at the interface of the intestinal immune system: target for immune modulation via dietary components. Eur J Pharmacol 2011;668(suppl 1):S124S132.

48 de Kivit S, Saeland E, Kraneveld AD, van de Kant HJ, Schouten B, van Esch BC, Knol J, Sprikkelman AB, van der Aa LB, Knippels LM, Garssen J, van Kooyk Y, Willemsen LE: Galectin- 9 induced by dietary synbiotics is involved in suppression of allergic symptoms in mice and humans. Allergy 2012;67:343-352. 